\title{
Study on the Validity of Surface Electromyography as Assessment Tools for Facial Nerve Palsy
}

\author{
Hye-Min Ryu', Seung-Jeong Lee', Eun-Jin Park', Su-Gyeong Kim', Kyeong Han \\ $\mathrm{Kim}^{2}$, Yoo Min $\mathrm{Choi}^{3}$, Jong Uk Kim³, Beom Yong Song ${ }^{3}$, Cheol Hong Kim', Hyun-Min \\ Yoon ${ }^{1 *}$, Tae-Han Yook ${ }^{3 *}$
}

1 Department of Acupuncture \& Moxibustion Medicine, College of Korean Medicine, Dong-Eui University, Busan, Korea

${ }^{2}$ Department of Preventive Medicine, College of Korean Medicine, Woosuk University, Jeonbuk, Korea

${ }^{3}$ Department of Acupuncture \& Moxibustion Medicine, College of Korean Medicine, Woosuk University, Jeonbuk, Korea

\section{Key Words}

surface electromyography (sEMG), facial nerve palsy, nerve conduction study (NCS), house-brackmann grade, yanagihara unweighted grading scale, sunnybrook facial grading system

\section{Abstract \\ Objectives: The purpose of this study was to find out validity of Surface Electromyography(sEMG) compared with Nerve Conduction Study and clinical assessment scale as assessment factors for facial palsy.}

Methods: We investigated 50 cases of patients with peripheral facial palsy who had records of sEMG and NCS to check. Then we analyzed the correlation between sEMG and NCS that carried out around 1 week after onset. And we analyzed the correlation between sEMG and clinical assessment scales that were measured three times around 1 week, 3-4 weeks and 5-6 weeks after onset. Clinical assessment scales used in this study were House-brackmann grade, Yanagihara unweighted grading scale and Sunnybrook facial grading system. We used Pearson's correlation for statistical analysis.

Received: Nov 01, 2018 Reviewed: Nov 02, 2018 Accepted: Nov 14, 2018

(c) This is an Open-Access article distributed under the terms of the Creative Common Attribution Non-Commercial License (http://creativecommons.org/licenses/by-nc/4.0/) which permits unrestricted noncommercial use, distribution, and reproduction in any medium, provided the original work is properly cited.

This paper meets the requirements of KS X ISO 9706, ISO 9706-1994 and ANSI/NISO Z39.48-1992 (Permanence of Paper).
Results: SEMG and NCS, measured at similar times, were statistically correlated. Especially, the correlation with the forehead region was high. And sEMG and clinical assessment scale, measured at same time, were statistically correlated, especially after 5 weeks from onset.

Conclusion: According to this study, sEMG is expected to be useful to assessment facial palsy.

\section{Introduction}

Facial nerve palsy is the most common disease of cranial nerve. The symptoms of eyes and mouth twisted to one side due to paralysis of the facial muscles are typical and It may be accompanied by abnormal sensation and pain of the face or the cervix, decreased tear, taste disorder, decreased saliva secretion, pain around the ear, and hearing sensitivity [1].

The assessment tools for facial nerve palsy include clinical assessment scales such as House-Brackmann grade, Yanagihara unweighted grading system, Sunnybrook facial grading system, which evaluate the paralysis by the evaluator by visually checking the appearance of the patient $[2,3]$, and electrophysical tests such as nerve excitability test (NET), Nerve conduction study (NCS), electromyography (EMG), blink reflex [4]. Among these, EMG is a method of quantifying the de-

*Corresponding Author

Hyun-Min Yoon. Department of Acupuncture \& Moxibustion Medicine, Dong-Eui University Hospital of Korean Medicine, San 45-1, Yangjung 2-Dong, Busanjin-Gu, Busan 614-710, Korea

Tel: +82-51-850-8934 Fax: +82-51-867-5162 E-mail: 3rdmed@hanmail.net

Tae-Han Yook. Department of Acupuncture \& Moxibustion Medicine, Woosuk Univer-

sity Hospital of Korean Medicine, 46 Eoeun-ro, Wansan-gu, Jeonju, Jeonbuk 560-833, Korea

Tel: +82-63-220-8625 Fax: +82-63-220-8400 E-mail: nasiss@naver.com 
gree of contraction of skeletal muscles, and EMG analysis has been actively used as an effective method for evaluating the contraction activity of human muscles [5].

EMG is an electrical measurement method for one unit of motion in an invasive manner using a needle electrode. This method causes pain to the patient and only detects information related to the tip of the needle electrode [6]. On the other hand, although surface electrodes have a disadvantage in having high cross noise in the signal being measured and measuring the activity of muscles located at the core, the patient has no pain and simple to the tester because of non-invasive method of attaching electrodes to the surface. And all information of one motion unit can be detected $[5,7]$.

Studies on the exercise and rehabilitation of the musculoskeletal system have been actively conducted using the characteristics of sEMG. There are studies on the use of sEMG in the facial muscles, such as the location of the electrodes $[8,9]$, the types of electrodes, Frigerio A et al. [10] Have studied the sEMG of the orbicularis oculi m. and subsequently, in Korea, Kim et al. studied the motion of the orbicularis oris $\mathrm{m}$. of health people. In a study for facial nerve palsy, Kim et al. [11] reported the correlation between sEMG and clinical assessment scale of facial nerve palsy in 21 patients within 90 days of onset. However, there was no follow-up study or comparison of sEMG with conventional electrophysiology.

Therefore, in this study, we compared the clinical records of NCS, sEMG, and clinical assessment scales in patients with facial nerve palsy between August 2017 and January 2018. The research was conducted.

\section{Methods}

\subsection{Patients}

We reviewed the medical records of patients who visited the OO University Korean Medicine Hospital who were diagnosed with peripheral facial nerve palsy, and who had received inpatient and outpatient treatment between October, 2017 and January, 2018. Approval for the study was received from the institutional review board of Dong-Eui University Korean Medicine Hospital (IRB no. 2016-08)

The inclusion criteria were: (1) patients were diagnosed with peripheral facial nerve palsy; (2) patients had presented to hospital within 14 days of the onset of facial nerve palsy; (3) patients had records of NCS and three sEMG to check.

The exclude criteria were: (1) patients had facial palsy caused by central nerve disease; (2) patients had history of facial palsy; (3) patients had facial skin disease; (4) patients had difficulty in moving the face by plastic surgery of facial surgery; (5) patients had systemic diseases that can affect facial sEMG; (6) patients were deemed inappropriate by researcher.

\subsection{Assessment scale}

We used the House-Brackmann grade, the Yanagihara unweighted grading system, and the Sunnybrook facial grading system as clinical assessment scale for facial nerve palsy.

\subsubsection{House-Brackmann grade (HBGS) [12]}

Depending on the degree of paralysis, G I to G VI are evaluated in six steps. G I means complete normal state, G VI means complete paralysis, and the more severe the paralysis, the larger the number.

\subsubsection{Yanagihara unweighted grading system (Y-Score) [13]}

The total score is calculated by imposing a score of $0-4$ points on each of 10 items evaluating the state and motion of each facial region. The complete paralysis is assessed as 0 point and the normal 4 point. The total score is 40 points. The more severe the paralysis, the lower the score.

\subsubsection{Sunnybrook facial grading system (S-Score) [14]}

It is method to evaluate the paralysis of the laralysis by subtracting score of Symmetry of resting multiplied 5 and score of synkinesis from score of Symmetry of voluntary movement multiplied 4 . The total score of the normal state is 100 points. The more severe the paralysis, the lower the score.

\subsection{Measurements}

\subsubsection{Measuring instrument}

We measured sEMG of patients with facial nerve palsy using QEMG-4XL (LAXRTHA, Korea). The sEMG was measured by attaching the disposable electrode to the surface of the muscle group and then connecting the ground electrode.

\subsubsection{Measuring method}

The sEMG was measured by a Korean medicine doctor who was fully familiar with the measurement method. Measurements were performed around a week after onset, around 3-4 weeks after the onset, and 5-6 weeks after the onset. The NCS was performed by the Department of Neurology at $\mathrm{OO}$ Hospital.

The sEMG measurement site was selected from the forehead, cheek and mouth, where muscle movement was significant and electrode attachment was relatively easy. Electrode Were attached to GB14 * middle of ST4 and SI18 - side 1 chon (寸) of the CV24, corresponding to Frontalis $\mathrm{m}$, Zygomaticus major m, Orbicularis oris m, such. Ground electrode were attached TE17 [15].

Before attaching the electrodes, the measurer will fully explain the movement of the muscles in a state of facing the patient and allow the patient to perform the movement of the muscles correctly. Frontalis m. was examined by raising eyebrows and making forehead wrinkles. Zygo- 
maticus major $\mathrm{m}$. was examined by raising the cheek. Orbicularis oris $\mathrm{m}$. was examined by making the lips round like when make an 'o' sound. Tension time keeps the muscles moving for 3 seconds and relaxation time keeps the muscles relaxed for 5 seconds. Tension time and relaxation time are repeated three times and the EMG of the muscle is measured. The examination time was about 20 minutes including preparation time and cleaning time.

The unit of measured value of sEMG is root mean square (RMS), which is the EMG signal amplitude value obtained by squaring signal magnitude for a certain time interval. In this study, the measurement value of sEMG, expressed as root mean square (RMS), was quantified as the ratio of the infected side to the unaffected side (\%). NCS records the Compound Muscle Action Potentials (CMAP), which occurs in the distal muscle of the nerve after giving maximum stimulus to the proximal portion of the peripheral nerve. Terminal latency, amplitude, and nerve conduction velocity (NCV) are measured by this method. Among the measurements of Orbicularis Oculi collected by NCS test, the amplitude value was quantified as the ratio of the affected side to the unaffected side (\%).

\subsection{Statistical analysis}

Statistical analysis was performed using SPSS 18.0 for Windows (SPSS Co, USA). Values were presented as mean \pm standard deviation (SD). Pearson's correlation analysis was used to analyze the correlation between sEMG, NCS, and clinical evaluation, and p-value less than 0.05 was considered significant. Correlation coefficient (r) was shown in Pearson correlation analysis results.

\section{Results}

\subsection{General characteristics}

The gender distribution of 50 subjects in this study was 28 men (56\%) and 22 women (44\%). The mean age was 49.56 \pm 14.04 years. The direction of paralysis was 32 cases $(64 \%)$ in the right side and 18 cases (36\%) in the left side. HBGS, measured around a week after onset, was III in 11 cases (22\%), IV in 12 cases (24\%), and V 27 in 27 cases (54\%). (Table 1)

The mean of the NCS measurements measured around a week after the onset was $56.04 \pm 18.66 \%$. The mean value of sEMG measurements in the frontalis region was $31.59 \pm$ $21.20 \%$ in the first, $62.42 \pm 48.21 \%$ in the second, and 65.32 $\pm 26.46 \%$ in the third. The mean value of sEMG in the Zygomaticus region was $40.68 \pm 23.22 \%$ in the first, $64.88 \pm$ $27.75 \%$ in the second, and $63.63 \pm 25.60 \%$ in the third. The mean value of Oribicularis oris was $40.44 \pm 22.29 \%$ in the first, $56.55 \pm 25.56 \%$ in the second, and $58.19 \pm 23.90 \%$ in the third. The mean value of HBGS was $4.32 \pm 0.82$ in the first, $3.12 \pm 1.08$ in the second, and $2.38 \pm 1.01$ in the third. The mean value of Y-sore was $13.1 \pm 6.07$ in the first, $26.12 \pm$ 8.93 in the second, and $31.42 \pm 7.78$ in the third. The mean value of S-score was $38.00 \pm 18.62$ in the first, $66.96 \pm 22.49$ in the second, and $78.97 \pm 19.08$ in the third. (Table 2)

\subsection{Correlations between NCS and sEMG}

We analyzed correlations between NCS and sEMG that were measured around 1 week after onset. There was a statistically significant positive correlation between the NCS and the sEMG measurements of the frontalis region, the Zygomaticus region, the mean of the three regions. And the sEMG measurement of the frontalis region was most correlated with the NCS measurement. (Table 3)

\subsection{Correlations between NCS, sEMG and Clinical scales}

\subsubsection{Around a week from onset}

There was a statistically significant negative correlation between the NCS measurement and HBGS. And threr was a statistically significant positive correlation between NCS measurement and Y-score, S-score. subsections of Y-score except yl. There was a statistically significant positive correlation between the sEMG measurement of frontalis region and Y-score, S-score, y2, y8-10. There was a statistically significant negative correlation between the SEMG measurement of zygomaticus region and HBGS. And there was a statistically significant positive correlation between the sEMG measurement of zygomaticus region and S-score, all subsections of Y-score. There was not a statistically significant correlation between the sEMG measurement of orbicularis oris region and all of clinical assessment scale. There was a statistically significant positive correlation between the sEMG measurement of orbicularis oris region and Y-score, S-score, y2, 3, 9, 10. (Table 4)

\subsubsection{Around 3-4 weeks after Onset}

There was a statistically significant positive correlation between the sEMG measurement of frontalis region and Y-score, S-score, y2 and y7-10. There was a statistically significant positive correlation between the sEMG measurement of zygomaticus region and Y-score, y2-4, y6 and y7. There was a statistically significant negative correlation between the sEMG measurement of orbicularis oris region and HBGS. And there was a statistically significant positive correlation between the sEMG measurement of orbicularis oris region and Y-score, S-score, all subsections of Y-score. There was a statistically significant negative correlation between the mean of sEMG measurement and HBGS. And there was a statistically significant positive correlation between the mean of sEMG measurement and Y-score, S-score, all subsections of Y-score. (Table 5)

\subsubsection{Around 5-6 weeks after Onset}

There was a statistically significant negative correlation between the sEMG measurements of 3 regions, the mean of sEMG measurement and HBGS each. And there was a statistically significant positive correlation between the sEMG measurements of 3 regions, the mean of sEMG measurement and Y-score, S-score, all subsections of Y-score, each. (Table 6) 
Table 1 Basic Characteristics

\begin{tabular}{cccc}
\hline Classification & & $\mathbf{N}$ & \% \\
\hline \multirow{2}{*}{ Sex } & Male & 28 & $56.0 \%$ \\
& Female & 22 & $44.0 \%$ \\
Site of facial palsy & Right & 32 & $64.0 \%$ \\
& Left & 18 & $36.0 \%$ \\
& III & 11 & $22.0 \%$ \\
HBGS & IV & 12 & $24.0 \%$ \\
& V & 27 & $54.0 \%$ \\
\hline
\end{tabular}

Table 2 Mean of NCS and sEMG Clinical Assessment Scale

\begin{tabular}{|c|c|c|c|c|c|c|c|}
\hline \multirow{2}{*}{\multicolumn{2}{|c|}{ Classification }} & \multicolumn{2}{|c|}{1 st } & \multicolumn{2}{|c|}{ 2nd } & \multicolumn{2}{|c|}{ 3rd } \\
\hline & & Mean (\%) & SD & Mean (\%) & SD & Mean (\%) & SD \\
\hline \multirow[t]{2}{*}{$\mathrm{NCS}$} & Orbicularis Oculi & 56.04 & 18.66 & - & - & - & - \\
\hline & Frontalis & 31.59 & 21.20 & 62.42 & 48.21 & 65.32 & 26.46 \\
\hline \multirow[t]{5}{*}{ sEMG } & Zygomaticus & 40.68 & 23.22 & 64.88 & 27.75 & 63.63 & 25.60 \\
\hline & Orbicularis oris & 40.44 & 22.29 & 56.55 & 25.56 & 58.19 & 23.90 \\
\hline & HBGS & 4.32 & 0.82 & 3.12 & 1.08 & 2.38 & 1.01 \\
\hline & Y-Score & 13.10 & 6.07 & 26.12 & 8.93 & 31.42 & 7.78 \\
\hline & S-Score & 38.00 & 18.62 & 66.96 & 22.49 & 79.78 & 19.08 \\
\hline
\end{tabular}

Table 3 Correlations between NCS and sEMG

\begin{tabular}{ccc}
\hline Classification & N & NCS \\
\hline F1 & 0.375 & $0.007^{*}$ \\
Z1 & 0.330 & $0.019^{*}$ \\
O1 & -0.040 & 0.784 \\
M1 & 0.351 & $0.012^{*}$ \\
\hline
\end{tabular}

F1 : The 1st sEMG measure of Frontalis $\mathrm{m}$.

$\mathrm{Z1}$ : The 1st sEMG measure of Zygomaticus $\mathrm{m}$.

O1 : The 1st sEMG measure of Orbicularis oirs $\mathrm{m}$.

M1 : Mean of 1st sEMG measures of 3 sites

* : p-value $<0.05$ by Pearson's correlation.

$\mathrm{r}$ : Pearson's correlation coefficient 
Table 4 Correlations between NCS, sEMG and Clinical Assessment Scales around 1 week after Onset

\begin{tabular}{ccccccccccc}
\hline \multirow{2}{*}{ Classification } & \multicolumn{2}{c}{ NCS } & \multicolumn{2}{c}{$\mathbf{F 1}$} & \multicolumn{2}{c}{$\mathbf{Z 1}$} & \multicolumn{2}{c}{ O1 } & \multicolumn{2}{c}{ M1 } \\
& $\mathbf{r}$ & $\mathbf{p}$ & $\mathbf{r}$ & $\mathbf{p}$ & $\mathbf{r}$ & $\mathbf{p}$ & $\mathbf{r}$ & $\mathbf{p}$ & $\mathbf{r}$ & $\mathbf{p}$ \\
\hline HBGS & -0.522 & $0.000^{*}$ & -0.180 & 0.211 & -0.329 & $0.020^{*}$ & -0.062 & 0.668 & -0.306 & $0.030^{*}$ \\
Y-score & 0.556 & $0.000^{*}$ & 0.293 & $0.039^{*}$ & 0.247 & 0.083 & 0.037 & 0.799 & 0.305 & $0.031^{*}$ \\
y1 & 0.270 & 0.058 & 0.056 & 0.701 & 0.167 & 0.248 & -0.155 & 0.281 & 0.038 & 0.794 \\
y2 & 0.454 & $0.001^{*}$ & 0.448 & $0.001^{*}$ & 0.187 & 0.192 & 0.054 & 0.708 & 0.359 & $0.010^{*}$ \\
y3 & 0.484 & $0.000^{*}$ & 0.228 & 0.112 & 0.278 & 0.050 & 0.025 & 0.865 & 0.283 & $0.047^{*}$ \\
y4 & 0.293 & $0.039^{*}$ & 0.154 & 0.287 & 0.154 & 0.286 & 0.090 & 0.553 & 0.211 & 0.141 \\
y5 & 0.388 & $0.005^{*}$ & 0.217 & 0.131 & 0.178 & 0.216 & 0.027 & 0.852 & 0.222 & 0.120 \\
y6 & 0.371 & $0.008^{*}$ & -0.041 & 0.777 & 0.158 & 0.273 & 0.003 & 0.985 & 0.068 & 0.637 \\
y7 & 0.357 & $0.011^{*}$ & 0.094 & 0.517 & 0.200 & 0.164 & -0.071 & 0.626 & 0.121 & 0.404 \\
y8 & 0.509 & $0.000^{*}$ & 0.329 & $0.020^{*}$ & 0.064 & 0.661 & 0.087 & 0.550 & 0.247 & 0.083 \\
y9 & 0.556 & $0.000^{*}$ & 0.411 & $0.003^{*}$ & 0.307 & $0.030^{*}$ & 0.105 & 0.469 & 0.434 & $0.002^{*}$ \\
y10 & 0.595 & $0.000^{*}$ & 0.295 & $0.038^{*}$ & 0.241 & 0.092 & 0.087 & 0.550 & 0.329 & $0.020^{*}$ \\
S-score & 0.627 & $0.000^{*}$ & 0.376 & $0.007^{*}$ & 0.298 & $0.036^{*}$ & 0.125 & 0.387 & 0.422 & $0.002^{*}$ \\
\hline
\end{tabular}

F1: The 1st sEMG measure of Frontalis $\mathrm{m}$.

$\mathrm{Z1}$ : The 1st sEMG measure of Zygomaticus $\mathrm{m}$.

O1 : The 1st sEMG measure of Orbicularis oirs $\mathrm{m}$.

M1 : Mean of 1st sEMG measures of 3 sites

y1-10 : subsections of Yanagihara unweighted grading system

* : p-value $<0.05$ by Pearson's correlation

r : Pearson's correlation coefficient 
Table 5 Correlations between NCS, sEMG and Clinical Assessment Scales around 3-4 weeks after Onset

\begin{tabular}{cccccccccccc}
\hline Classification & \multicolumn{2}{c}{$\mathbf{F} 2$} & \multicolumn{2}{c}{$\mathbf{Z} 2$} & \multicolumn{2}{c}{$\mathbf{O 2}$} & \multicolumn{2}{c}{ M2 } & \multicolumn{3}{c}{ M1 } \\
& $\mathbf{r}$ & $\mathbf{p}$ & $\mathbf{r}$ & $\mathbf{p}$ & $\mathbf{r}$ & $\mathbf{p}$ & $\mathbf{r} 7$ & $\mathbf{p}$ & $\mathbf{r}$ & $\mathbf{p}$ \\
\hline HBGS & -0.270 & 0.058 & -0.192 & 0.181 & -0.620 & $0.000^{*}$ & -0.491 & $0.000^{*}$ & -0.306 & $0.030^{*}$ \\
Y-score & 0.311 & $0.028^{*}$ & 0.308 & $0.030^{*}$ & 0.659 & $0.000^{*}$ & 0.579 & $0.000^{*}$ & 0.305 & $0.031^{*}$ \\
y1 & 0.187 & 0.194 & 0.202 & 0.160 & 0.486 & $0.000^{*}$ & 0.388 & $0.005^{*}$ & 0.038 & 0.794 \\
y2 & 0.310 & $0.028^{*}$ & 0.301 & $0.034^{*}$ & 0.618 & $0.000^{*}$ & 0.561 & $0.000^{*}$ & 0.359 & $0.010^{*}$ \\
y3 & 0.241 & 0.092 & 0.372 & $0.008^{*}$ & 0.570 & $0.000^{*}$ & 0.524 & $0.000^{*}$ & 0.283 & $0.047^{*}$ \\
y4 & 0.235 & 0.100 & 0.323 & $0.022^{*}$ & 0.468 & $0.001^{*}$ & 0.463 & $0.001 *$ & 0.211 & 0.141 \\
y5 & 0.251 & 0.079 & 0.256 & 0.073 & 0.621 & $0.000^{*}$ & 0.503 & $0.000^{*}$ & 0.222 & 0.120 \\
y6 & 0.194 & 0.178 & 0.298 & $0.035^{*}$ & 0.545 & $0.000^{*}$ & 0.452 & $0.001 *$ & 0.068 & 0.637 \\
y7 & 0.280 & $0.049^{*}$ & 0.303 & $0.032^{*}$ & 0.582 & $0.000^{*}$ & 0.528 & $0.000^{*}$ & 0.121 & 0.404 \\
y8 & 0.332 & $0.018^{*}$ & 0.264 & 0.064 & 0.614 & $0.000^{*}$ & 0.560 & $0.000^{*}$ & 0.247 & 0.083 \\
y9 & 0.357 & $0.011^{*}$ & 0.218 & 0.129 & 0.671 & $0.000^{*}$ & 0.579 & $0.000^{*}$ & 0.434 & $0.002^{*}$ \\
y10 & 0.350 & $0.013^{*}$ & 0.200 & 0.163 & 0.674 & $0.000^{*}$ & 0.569 & $0.000^{*}$ & 0.329 & $0.020^{*}$ \\
S-score & 0.351 & $0.012^{*}$ & 0.264 & 0.064 & 0.702 & $0.000^{*}$ & 0.606 & $0.000^{*}$ & 0.422 & $0.002^{*}$ \\
\hline
\end{tabular}

F2 : The 2nd sEMG measure of Frontalis $\mathrm{m}$.

$\mathrm{Z2}$ : The 2nd sEMG measure of Zygomaticus $\mathrm{m}$.

O2 : The 2nd sEMG measure of Orbicularis oirs $\mathrm{m}$.

M2 : Mean of 2nd sEMG measures of 3 sites

y1-10 : subsections of Yanagihara unweighted grading system

* : p-value $<0.05$ by Pearson's correlation

$\mathrm{r}$ : Pearson's correlation coefficient 
Table 6 Correlations between NCS, sEMG and Clinical Assessment Scales around 5-6 weeks after Onset

\begin{tabular}{cccccccccccc}
\hline Classification & \multicolumn{2}{c}{$\mathbf{F 3}$} & \multicolumn{2}{c}{$\mathbf{Z 3}$} & \multicolumn{2}{c}{ O3 } & \multicolumn{2}{c}{ M3 } & \multicolumn{2}{c}{ M1 } \\
& r & $\mathbf{p}$ & $\mathbf{r}$ & $\mathbf{p}$ & $\mathbf{r}$ & $\mathbf{p}$ & $\mathbf{r}$ & $\mathbf{p}$ & $\mathbf{r}$ & $\mathbf{p}$ \\
\hline HBGS & -0.595 & $0.000^{*}$ & -0.424 & $0.002^{*}$ & -0.540 & $0.000^{*}$ & -0.658 & $0.000^{*}$ & -0.306 & $0.030^{*}$ \\
Y-Score & 0.669 & $0.000^{*}$ & 0.614 & $0.000^{*}$ & 0.555 & $0.000^{*}$ & 0.778 & $0.000^{*}$ & 0.305 & $0.031^{*}$ \\
y1 & 0.437 & $0.001^{*}$ & 0.437 & $0.002^{*}$ & 0.387 & $0.005^{*}$ & 0.533 & $0.000^{*}$ & 0.038 & 0.794 \\
y2 & 0.687 & $0.000^{*}$ & 0.392 & $0.005^{*}$ & 0.571 & $0.000^{*}$ & 0.697 & $0.000^{*}$ & 0.359 & $0.010^{*}$ \\
y3 & 0.548 & $0.000^{*}$ & 0.531 & $0.000^{*}$ & 0.471 & $0.000^{*}$ & 0.655 & $0.000^{*}$ & 0.283 & $0.047^{*}$ \\
y4 & 0.567 & $0.000^{*}$ & 0.582 & $0.000^{*}$ & 0.432 & $0.001^{*}$ & 0.670 & $0.000^{*}$ & 0.211 & 0.141 \\
y5 & 0.563 & $0.000^{*}$ & 0.566 & $0.000^{*}$ & 0.512 & $0.000^{*}$ & 0.694 & $0.000^{*}$ & 0.222 & 0.120 \\
y6 & 0.534 & $0.000^{*}$ & 0.619 & $0.000^{*}$ & 0.397 & $0.003^{*}$ & 0.657 & $0.000^{*}$ & 0.068 & 0.637 \\
y7 & 0.601 & $0.000^{*}$ & 0.570 & $0.000^{*}$ & 0.532 & $0.000^{*}$ & 0.720 & $0.000^{*}$ & 0.121 & 0.404 \\
y8 & 0.629 & $0.000^{*}$ & 0.580 & $0.000^{*}$ & 0.511 & $0.000^{*}$ & 0.728 & $0.000^{*}$ & 0.247 & 0.083 \\
y9 & 0.637 & $0.000^{*}$ & 0.564 & $0.000^{*}$ & 0.516 & $0.000^{*}$ & 0.727 & $0.000^{*}$ & 0.434 & $0.002^{*}$ \\
y10 & 0.691 & $0.000^{*}$ & 0.564 & $0.000^{*}$ & 0.589 & $0.000^{*}$ & 0.780 & $0.000^{*}$ & 0.329 & $0.020^{*}$ \\
S-score & 0.687 & $0.000^{*}$ & 0.576 & $0.000^{*}$ & 0.592 & $0.000^{*}$ & 0.784 & $0.000^{*}$ & 0.422 & $0.002^{*}$ \\
\hline
\end{tabular}

F3 : The 3rd sEMG measure of Frontalis $\mathrm{m}$.

Z3 : The 3rd sEMG measure of Zygomaticus $\mathrm{m}$.

O3 : The 3rd sEMG measure of Orbicularis oirs $\mathrm{m}$.

M3 : Mean of 3rd sEMG measures of 3 sites

y1-10 : subsections of Yanagihara unweighted grading system.

* : p-value $<0.05$ by Pearson's correlation.

$\mathrm{r}$ : Pearson's correlation coefficient 


\section{Discussion}

Facial nerve palsy is reported to occur in 20-30 people per 100,000 people [1]. And it occurs in all ages, but it is common in the 20-30s. The male to female ratio is the same and mostly unilateral, with the same on both sides. Facial nerve palsy is based on electrical nerve damage and degeneration, and $86 \%$ is reported to be fully recovered if degenerative changes do not occur [1]. Therefore, initial evaluation of facial nerve palsy is important for the treatment and prognosis evaluation, and an objective evaluation tool for the evaluation of the treatment results is needed.

The electro-diagnostic test can measure the facial nerve status, degree of impairment, and prognosis of facial nerve palsy in a relatively short period, and it was easy to track and observe the prognosis of a patient with persistent palsy. In the assessment of prognosis and report of treatment effect, it was necessary to use different assessment methods appropriately according to the treatment period, as Gantz et al. reported that the combination of NCS and EMG can distinguish between short-term recovery or long-term sequelae in patients with facial nerve palsy.

NCS is widely used to determine the status and prognosis of peripheral nerve disorders, so NCS is widely used to evaluate facial nerve palsy. NCS is used to diagnose and evaluate diseases by evaluating the functional status of the nerves through nerve activity potentials induced by stimulation of peripheral motor or sensory nerves. NCS shows changes in the amplitude and nerve conduction of the nerve activity potential in the muscles dominated by the denatured nerve [16].

The nEMG measures the potential during the resting period and during the voluntary contraction period by inserting the fine needle electrode into the muscle, so it has the advantage of being able to find activity units that are not found in other tests [17]. Grosheva et al. [18] Reported that nEMG was useful in assessing the prognosis of acute facial nerve palsy in a series of tests, and 14 days of onset were the appropriate time for testing because the neurological damage site can be identified after 14 days after the onset. However, nEMG causes pain in the patient due to the insertion of the electrode, and can be erroneous if not inserted correctly in a specific muscle [6].

In contrast, there is no pain induction, and the method is simpler than the conventional nEMG, which makes it easy to repeat measurement and follow-up because sEMG is a non-invasive method that attaches electrodes to the skin surface [5, 6, 8]. Nikolaus et al. [19] Have proposed a new direction of facial sEMG studies by studying the facial EMG patterns of normal people using the sEMG devices, Kim et al. [10] Suggested the possibility of sEMG as an assessment tool of facial nerve palsy by analyzing the correlation between sEMG and clinical assessment scale.

Although there have been studies to utilize sEMG for facial nerve palsy, there have been no studies that have obtained objective validity, and there have been no follow-up studies based on comparison with conventional electrophysiological methods or treatment timing. The aim of this study was to analyze the correlation between the sEMG and clinincal assessment scale according to treatment timing, NCS, which are widely used in the assessment of facial nerve palsy, to evaluate the objectivity of sEMG as an assessment tool for facial nerve palsy. This study was designed to analyze the patient's medical records retrospectively, which can check the records of sEMG, NCV, and clinical assessment scales among the facial nerve palsy patients treated at $\mathrm{OO}$ University Korean Medicine Hospital from August 1, 17 to February 15,

Clinical assessment scales were evaluated using House-Brackmann grade (HBGS), Yanagihara unweighted grading system (Y-score) and Sunnybrook facial grading system (S-score) on the day of measuring sEMG. NCS was measured around 1 week after the onset, and the amplitude value of Orbicularis oculi m. was quantified as the ratio of the affected side to the unaffected side (\%). sEMG was measured three times, around 1 week, 3-4weeks, 5-6weeks after the onset. The sEMG was measured on the forehead, cheek and mouth. Electrodes were attached at GB14, the middle ST4 and SI18 and on the side of CV24, corresponding to Frontali m., Zygomaticus major m., Orbicularis oris $\mathrm{m}$. The measurement value of sEMG, expressed as root mean square (RMS), was quantified as the ratio of the infected side to the unaffected side (\%).

As shown in this study, there was a significant correlation between NCS and sEMG measurement, and there was a significant correlation between sEMG and clinical assessment scale. There was a statistically significant positive correlation between the NCS and the sEMG measurements of the frontalis region, the Zygomaticus region, the mean of the three regions. And the sEMG measurement of the frontalis region was most correlated with the NCS measurement. The correlation between NCS and clinical assessment scale has already been revealed. In this study, there was a significant correlation with all clinical assessment scales except yl corresponding to asymmetry at rest, which is subsection of Y-score. HBGS had a significant negative correlation with all measured values except the sEMG measurements of the frontalis, orbicularis oris measured around lweek after onset, and the frontalis, zygomaticus measured around 3-4 weeks after onset. There was a significant positive correlation between the Y-score and the sEMG measurement except the measurements of zygomaticus and orbicularis oris around 1 week after the onset of the disease. Of the Y-score subsections, y2, related to forehead region, was significantly correlated with the sEMG measurements of the frontalis region at all period, and y8-10, related to mouth region, were significantly correlated with the sEMG measurements of the orbicularis oris region after 3 weeks after onset. S-score was significantly correlated with sEMG measurements except for the orbicularis oris region around 1 week after the onset.

These results indicate that the sEMG measured around 1 week after the onset was correlated with the NCS measurement used to evaluate facial nerve palsy, and there was a significant correlation between the sEMG measurement and the clinical assessment scale that externally assessed the status of facial palsy. Especially, the sEMG measurement after 5 weeks from onset had a significant correlation with the clinical assessment scales. This suggests that sEMG measurements can be used as an objective assessment tool for facial nerve palsy. The sEMG measurement of the orbicularis oris region around 1 week after the onset 
was not significantly correlated with the NCS measurement and all the clinical assessment scales. It was presumed that Orbicularis oris $\mathrm{m}$. was the muscle connected to the left and right, so movement of unaffected side effects the outcome. After 3 weeks, the paralysis was recovered and the affection of the normal side was considered to be low. However further study is necessary for clarifying the reasons and the drawbacks. In addition, the sEMG measurement of the frontalis region of the first test, the frontalis region, the zygomaitcus region of the second test were not correlated with the HBGS, and only the correlation with the Y-score was confirmed. This suggests that the Y-score can evaluate various paralysis compared to the HBGS divided 6 grade, because Y- score can be expressed by dividing the degree of the whole paralysis into a continuous number $(0-40)$. In the study of Kim et al., to evaluate facial nerve palsy, it was necessary to use a gross scale like HBGS and a regional grading system such as Y-system, because HBGS was general in each grade, it was difficult to express minute changes and it could not evaluate various degrees of facial paralysis. In the study results, the differences between measurement in the muscle group seem to be related to the characteristics of the sEMG, that the signals from the muscles around the measurement site are likely to affect the measurement results. In addition, unlike mechanical measurements, the clinical assessment scale may reflect some of the subjectivity of the examiner, so it was possible that an evaluation by another evaluator has caused an error. In order to utilize sEMG for facial nerve palsy, it was necessary to carry out studies to supplement this part, and it was expected that sEMG could be used for evaluation of facial nerve palsy through continuous research.

\section{Conclusion}

We studied 50 patients who were able to check NCS and sEMGs of patients who were treated with peripheral facial nerve palsy at $\mathrm{OO}$ University Korean Medicine Hospital from August 1, 2017 to February 15, 2018, and obtained the following results:

(1) There was a significant positive correlation between the NCS and measurement of sEMG at Frontalis and Zygomaticus region, the mean value of sEMG.

(2) There was a significant negative correlation between sEMG and HBGS, and there was a significant positive correlation with Y-score and S-score.

(3) There was a significant correlation in some cases, between the sEMG measurement and the clinical assessment scale around lweek after onset.

(4) There was a significant correlation in some cases, between the sEMG measurement at frontalis, zygomaticus region and the clinical assessment scale around 3-4 weeks after onset. There was a significant correlation between the sEMG measurement at orbicularis oris region and the clinical assessment scale around 3-4 weeks after onset.

(5) There was a significant correlation between sEMG measurement at all sites and clinical assessment scale around 5-6 weeks after onset.

\section{Acknowledgment}

This work was supported by the Ministry of Health and Welfare through the Korea Health Industry Development Institute(HB16C0028).

\section{Conflict of interest}

The authors declare that there are no conflicts of interest.

\section{References}

1. Korean Acupuncture \& Moxibustion Medicine Society. The Acupuncture and Moxibustion Medicine. Vol 1. Seiul : Hanmibook. 2016;638-642.

2. Kim MB, Kim JH, Shin SH, Yoon HJ, Ko WS. A study of facial nerve grading system. The Journal of Korean Oriental Medical Ophthalmology \& Otolaryngology \& Dermatology. 2007;20(3):147-60.

3. Lee JW, Kwon SA, Kim MJ, et al. A Study of Facial Palsy Sequelae and Evaluating Scale. The Journal of Korean Acupuncture \& Moxibustion Society. 2011;28(2):75-87.

4. Koo GH. Facial Nerve Palsy. Korean journal of pain. 1996;9(1):14-5.

5. Kim TW, Gong SJ, Gil SG, et al. Electromyographic analysis : theory and application. Seoul : Hanmi Med. 2013;28-43.

6. Choi YM. A study on the Measurement Method of Facial Surface Electromyograaphy at Buccal Area. Graduate School of Woosuk University; 2016.

7. Kim JY, Kim JU, et al. A Study of Surface Electromyography Measurement of Orbicularis oris motion in Healthy People. The Acupuncture. 2016;33(4):93-100.

8. Kim SH, Lee HY, Son DI, Jung CK, Ko DY. A Study on the Estimation of Motor Unit Information using Surface EMG. The Transactions of the Korean Institute of Electrical Engineers. 2007;56(11):2040-50.

9. Frigerio A, Cavallari P, Frigeni M, Pedrocchi A, Sarasola A, Ferrante S. Surface Electromyographic Mapping of the Orbicularis Oculi Muscle for Real-Time Blink Detection. JAMA Facial Plast Surg. 2014;16(5):335-42.

10. Kim JU, Lee HG, Jung DJ, et al. A Study on the Correlation between Surface Electromyography and Assessment Scale for Facial Palsy. The Acupunct. 2013;30(5):107-16.

11. Lee HG, IM JG, Jung DJ, Kim JU, Kim LH, Yook TH. Comparative Review on Oriental Medicine Study Utilized Surface Electromyography in Korea,China, Japan. Journal of Korean Acupuncture \& Moxibustion Medicine Society. 2013;30(1):23-34.

12. Okada T, Sakai T, Yoshida M, Kimura S. Electro-acupuncture for Facial Palsy with Synkinesis. Journal of the Japan Society of Acupuncture and Moxibustion. 2005;55(4):601-7.

13. House JW, Brackmann DE. Facial nerve grading systems. Otolaryngol Head Neck Sug. 1985;93:146-7.

14. Yanagihara N. Grading of Facial Palsy. In Facial Nerve Surgery, Proceedings: Third International Symposium of Facial Nerve Surgery, Zurich, 1976. U. Fisch(Ed). Amstelveen, Netherlands : Kugler Medical Publications. Birmingam, Al : Aesculapius Publishing Co. 1977;533-5. 
15. Ross BG, Fradet G, Nedzelskic JM. Development of a sensitive clinical facial grading system. Otolaryngol Head and Neck Surg. 1996;114:380-6.

16. Kim HB, Park YH, Bae SS. Clinical Application of Electromyography and Nerve Conduction Study. The Journal of Korean Society of Physical Therapy. 1998;10(1):199-212.

17. Sung WS, Baek YH, Kim PK, et al. Study on the Validity of Electromyography(EMG) And Early-performed Electroneurography(ENoG) as Predicting Factor for Facial Palsy - based on Collaborative Treatment between Korean and Western Medicine. The Acupuncture. 2013;30(4):115-123.

18. Grosheva M, Wittekindt C, Guntinas-Lichius O. Prognostic value of Electroneurography and Electromyography in facial palsy. Laryngoscope. 2008;118(3):394-7.

19. Schumann NP, Bongers K, Guntinas-Lichius O, Scholle HC. Facial muscle activation patterns in healthy male humans: A multi-channel surface EMG study. Journal of Neuroscience Methods. 2010;187(1):120-8. 